\title{
Dynamical Evolution of the Mass Function of the Galactic Globular Cluster System
}

\author{
Jihye Shin ${ }^{1}$, Sungsoo S. Kim ${ }^{1} \&$ Koji Takahashi ${ }^{2}$ \\ ${ }^{1}$ Dept. of Astronomy and Space Science, Kyung Hee University, Korea \\ email: jhshin@ap1.khu.ac.kr \\ ${ }^{2}$ Dept. of Informational Society Studies, Saitama Institude of Technology, Japan
}

\begin{abstract}
Using the most advanced anisotropic (2D) Fokker-Planck (FP) models, we calculate the evolution of the mass functions of the Galactic globular cluster system (GCMF). Our models include two-body relaxation, binary heating, tidal shocks, dynamical friction, stellar evolution, and realistic cluster orbits. We perform 2D-FP simulations for a large number of virtual globular clusters and synthesize these results to study the relation between the initial and present GCMFs. We found two probable IGCMFs that eventually evolve into the Milky Way GCMF : truncated power-law, and log-normal model with higher initial low mass limit and peak mass than the earlier studies.
\end{abstract}

Keywords. Globular Clusters, Fokker-Planck method.

\section{Introduction}

There have been many studies on the GCMF with various simulation methods. Vesperini (1997), Vesperini \& Heggie (1997), Vesperini (1998), Baumgardt (1998), and Baumgardt \& Makino (2003) used an N-body method, Okazaki \& Tosa (1995), Ostriker \& Gnedin (1997), Vesperini (1997), and Fall \& Zhang (2001) used simple analytical models, and Murali \& Weinberg (1997a), Murali \& Weinberg (1997b), and Murali \& Weinberg (1997c) used an isotropic FP model. However, not all of the the related physics (i.e., tidal shocks, realistic orbits, dynamical friction, stellar evolution, and a wide range of cluster parameters) were considered by any of the previous studies.

To consider all of the related physics for the evolution of the star cluster, we use the most advanced 2D-FP developed by Takahashi \& Lee (2000). The main difference of our calculation from the past studies is the realistic cluster orbit moving in the axisymmetric Galactic potential with eccentricities. We adjust the magnitude of the tidal shocks and dynamical friction with time according to the realistic velocity and galactocentric distance. Thanks to relatively short computing times of 2D-FP, we perform a total of 578 simulations with various initial conditions.

\section{Results}

The two-body relaxation causes the low mass part of the GCMF to decrease faster, and this changes the shape of GCMF to be log-normal even if the initial GCMF (IGCMF) is a power-low function. The contribution of the external effects on the GCMF is found to be more important than in earlier studies, and this is mainly because the realistic orbits considered by us result in faster cluster evolution. This generally causes our peak cluster masses smaller than earlier studies.

The IGCMFs that best fit the Milky Way GCMF at $12 \mathrm{Gyr}$ are a power-law IGCMF with $M_{\text {low }}=10^{6} M_{\odot}$ and a log-normal IGCMF with $M_{\text {peak }}=10^{6} M_{\odot}\left(M_{\text {low }}\right.$ is the lower 

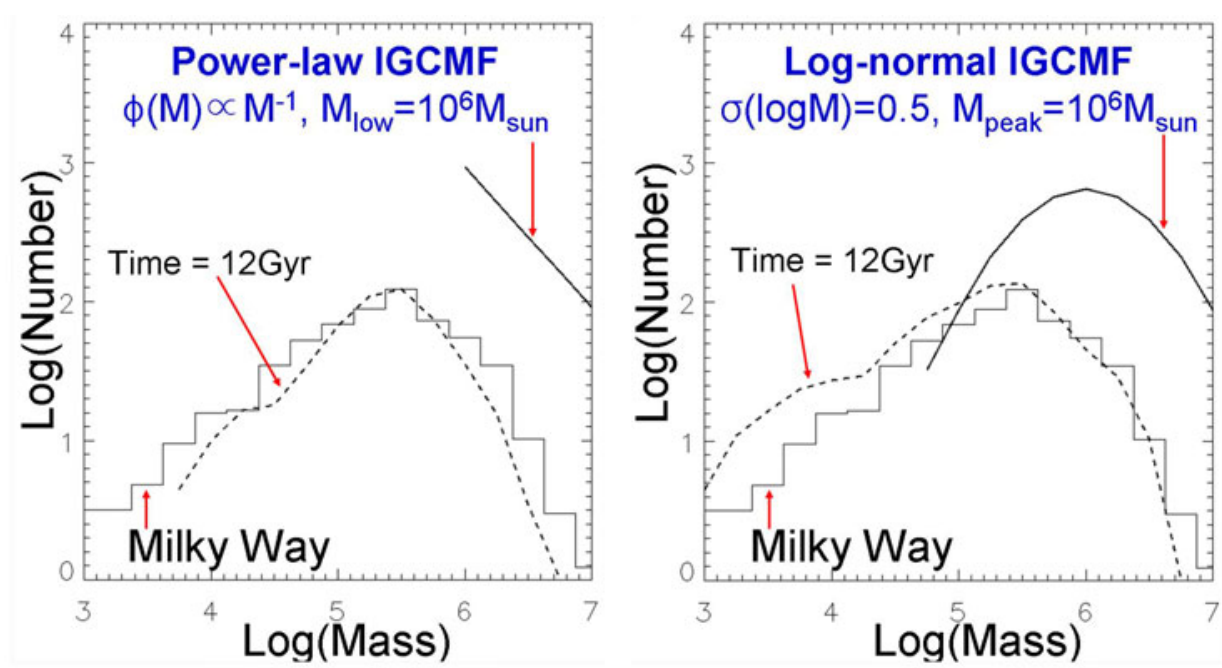

Figure 1. Two probable initial globular cluster mass functions that eventually evolve into the Milky Way GCMF.

cluster mass cutoff and $M_{\text {peak }}$ is the peak cluster mass). These IGCMFs have higher initial $M_{\text {low }}$ or $M_{\text {peak }}$ than the earlier studies. This is caused by the contribution of external effects that effectively shift the overall location of GCMF to the lower mass side. We find that the shape of the GCMF is mainly determined by the massive clusters with $10^{6} M_{\odot} \leqslant M \leqslant 10^{7} M_{\odot}$. In spite of the good fit to the Milky Way GCMF, the feasibility of the power-law IGCMF truncated at $10^{6} M_{\odot}$ is somewhat questionable because there is no proper theoretical base for such a high $M_{\text {low }}$. Therefore, we propose the log-normal model with $M_{\text {peak }}=10^{6} M_{\odot}$ as a best-fit IGCMF for the Milky Way. Log-normal IGCMFs could be naturally generated by expulsion from the proto-cluster of star forming gas due to supernova activity (Parmentier \& Gilmore 2007).

\section{References}

Baumgardt, H. 1998, A\&A, 330, 480

Baumgardt, H. \& Makino, J. 2003, MNRAS, 340, 227

Fall, S. \& Zhang, Q. 2001, ApJ, 561, 751

Gnedin, O. Y., Hernquist, L., \& Ostriker, J. P. 1999, ApJ, 514, 109

Gnedin, O. Y., Lee, H. M., \& Ostriker, J. P. 1999, ApJ, 522, 935

Murali, C. \& Weinberg, M. D. 1997a, MNRAS, 288, 749

Murali, C. \& Weinberg, M. D. 1997b, MNRAS, 288, 767

Murali, C. \& Weinberg, M. D. 1997c, MNRAS, 291, 717

Okazaki, T. \& Tosa, M. 1995, MNRAS, 274, 48

Ostriker, J. P. \& Gnedin, O. Y. 1997, ApJ, 474, 223

Parmentier, G. \& Gilmore, G. 2007, MNRAS, 377, 352

Takahashi, K. \& Lee, H. M. 2000 MNRAS, 316, 671

Vesperini, E. 1997 MNRAS, 287, 915

Vesperini, E. \& Heggie, D. C. 1997 MNRAS, 289, 898

Vesperini, E 1998 MNRAS, 299, 1019 